\title{
BMJ Open Protocol for the ProCare Trial: a phase II randomised controlled trial of shared care for follow-up of men with prostate cancer
}

\author{
Jon Emery, ${ }^{1,2}$ Juanita Doorey, ${ }^{2}$ Michael Jefford, ${ }^{3}$ Madeleine King, ${ }^{4}$ Marie Pirotta, ${ }^{1}$ \\ Dickon Hayne, ${ }^{5,6}$ Andrew Martin, ${ }^{7}$ Lyndal Trevena, ${ }^{8}$ Tee Lim, ${ }^{9}$ Roger Constable, ${ }^{10}$ \\ Cynthia Hawks, ${ }^{6}$ Amelia Hyatt, ${ }^{11}$ Akhlil Hamid, ${ }^{12}$ John Violet, ${ }^{13}$ Suki Gill, ${ }^{13}$ \\ Mark Frydenberg, ${ }^{14}$ Penelope Schofield ${ }^{11}$
}

To cite: Emery J, Doorey J, Jefford M, et al. Protocol for the ProCare Trial: a phase II randomised controlled trial of shared care for follow-up of men with prostate cancer BMJ Open 2014;4:e004972. doi:10.1136/bmjopen-2014004972

- Prepublication history for this paper is available online. To view these files please visit the journal online (http://dx.doi.org/10.1136/ bmjopen-2014-004972).

Received 31 January 2014 Revised 10 February 2014 Accepted 11 February 2014

CrossMark

For numbered affiliations see end of article.

Correspondence to Professor Jon Emery; jon.emery@unimelb.edu.au

\section{ABSTRACT}

Introduction: Men with prostate cancer require longterm follow-up to monitor disease progression and manage common adverse physical and psychosocial consequences of treatment. There is growing recognition of the potential role of primary care in cancer follow-up. This paper describes the protocol for a phase II multisite randomised controlled trial of a novel model of shared care for the follow-up of men after completing treatment for low-moderate risk prostate cancer.

Methods and analysis: The intervention is a shared care model of follow-up visits in the first 12 months after completing treatment for prostate cancer with the following specific components: a survivorship care plan, general practitioner (GP) management guidelines, register and recall systems, screening for distress and unmet needs and patient information resources. Eligible men will have completed surgery and/or radiotherapy for lowmoderate risk prostate cancer within the previous 8 weeks and have a GP who consents to participate. Ninety men will be randomised to the intervention or current hospital follow-up care. Study outcome measures will be collected at baseline, 3, 6 and 12 months and include anxiety, depression, unmet needs, prostate cancer-specific quality of life and satisfaction with care. Clinical processes and healthcare resource usage will also be measured. The principal emphasis of the analysis will be on obtaining estimates of the treatment effect size and assessing feasibility in order to inform the design of a subsequent phase III trial.

Ethics and dissemination: Ethics approval has been granted by the University of Western Australia and from all hospital recruitment sites in Western Australia and Victoria.

Results: of this phase II trial will be reported in peer-reviewed publications and in conference presentations.

Trial Registration: Australian New Zealand Clinical Trial Registry ACTRN12610000938000

\section{Strengths and limitations of study}

- This is the first randomised controlled trial of a model of shared care for men with prostate cancer.

- It is also the first trial to use the distress thermometer in primary care and the first to test a specific checklist to identify unmet needs of cancer survivors in primary care.

- As a phase II trial of a complex intervention it is designed to provide preliminary estimates of the feasibility and the efficacy of the shared care intervention for phase III planning purposes.

\section{INTRODUCTION}

Prostate cancer is the second most common cause of cancer among men worldwide, with the highest estimated incidence rates being in Australia and New Zealand, north America and western and northern Europe. ${ }^{1}$ Age-standardised incidence rates in 2008/ 100000 men were 104.2 in Australia and New Zealand; 93.1 in western Europe and 85.6 in northern America. $^{2}$ In Australia 19438 men were newly diagnosed with prostate cancer in $2009^{3}$ and the incidence is projected to increase to approximately 25310 by $2020 .{ }^{4}$ In the USA, there were an estimated 246000 new prostate cancer cases in 2010 and this is projected to rise to 322000 by $2020 .^{5}$ These changes in prostate cancer incidence are largely due to the growing use of the prostate-specific antigen (PSA) as a screening test but also due to the ageing population. ${ }^{3}$

Although prostate cancer is a common cause of death from cancer, a 5-year survival is relatively high. Between 2006 and 2010, the 5-year relative survival rate for men 
diagnosed with prostate cancer in Australia was 92\%, with survival being the highest for men aged 5069 years. ${ }^{3}$ Most recent data from the USA show a 5-year relative survival for prostate cancer of $99 \%{ }^{6}$ Men who have completed treatment for prostate cancer require long-term follow-up, to detect recurrence or progression of the disease, monitor any adverse effects of treatment and to identify and address any ongoing psychosocial needs. ${ }^{7}$ Men with prostate cancer also frequently have a range of comorbid conditions requiring management.

\section{Prostate cancer: high burden of illness}

Observational studies from the USA and the UK have demonstrated that men treated for prostate cancer frequently experience distressing and ongoing side effects, most notably urinary and bowel incontinence, sexual dysfunction and significant psychological issues. ${ }^{8} 9$ The severity and duration of side effects vary by treatment modality. A recently published study from the USA of 1655 men treated for localised prostate cancer with a 15-year follow-up found that men having a prostatectomy were more likely to have urinary incontinence and erectile dysfunction at 2 and 5 years post-treatment than those undergoing radiotherapy, but less likely to have bowel urgency. ${ }^{10}$

Research also demonstrates that following prostate cancer treatment the majority of men have unmet psychological and supportive care needs. A cross sectional survey of 1001 men with prostate cancer living in seven European countries found that $81 \%$ had some unmet supportive care needs, including psychological, sexual and health system and information needs. ${ }^{11}$ In a population-based cohort of 978 Australian men with recently treated prostate cancer, $54 \%$ had unmet psychological needs, particularly 'uncertainty about the future' $(21 \%)$ and $47 \%$ unmet sexual needs. ${ }^{12} \mathrm{~A}$ larger Australian longitudinal study with a 3-year follow-up compared men treated for prostate cancer with matched controls to account for potential effects of normal ageing. Men treated for prostate cancer had lower sexual function, especially those on androgen deprivation therapy (97\% impotence at 3 years), compared with 53\% under active surveillance. ${ }^{13}$ At 3 years, $67.9 \%$ of men who had nerve sparing radical prostatectomy and $86.7 \%$ of men who had non-nerve sparing radical prostatectomy were impotent. Men treated with radical prostatectomy reported worst urinary function $(16 \%$ incontinence at 1 year, $12 \%$ at 3 years) compared with $3 \%$ incontinence after 3 years in the active surveillance group; and bowel function was worst in those receiving external beam radiotherapy (15\% moderate or severe bowel problems at 3 years; compared with $3 \%$ after 3 years of active surveillance.)

\section{Current care of men with prostate cancer in general practice}

The role of general practitioners (GPs) in prostate cancer screening is well recognised. General practice is also heavily engaged in managing men with prostate cancer including long-term treatment and related health problems. Longitudinal data from the UK on nearly 5000 survivors with prostate cancer (5 years or longer post diagnosis) found that these men consulted their GP up to three more times annually compared to controls, a trend that continued even 15 years after diagnosis. ${ }^{14}$ Compared to matched controls, prostate cancer survivors had 39\% more consultations over a 3-year follow-up period, partly due to monitoring and administration of hormonal treatments. Data from the Netherlands also showed that patients with prostate cancer consult their GP more than controls at 2-5 years after diagnosis, for both cancer-related health problems and chronic disease management. ${ }^{15}$

In Australia, data from the Bettering the Evaluation and Care of Health (BEACH) study showed that, of 2385 general practice consultations about prostate cancer in 2008 only $9 \%$ were for prostate cancer as a new problem (The BEACH Project: Bettering the Evaluation and Care of Health Australian General Practice Statistics and Classification Centre, 2009, personal communication). The following services were provided: PSA test request $(21 \%)$; counselling, advice and education $(15 \%)$; local injection/implant insertion (12\%); prescription, predominantly for opioids and antiandrogens (approximately $30 \%$ ); and referral, predominantly to urology or oncology (10\%).

An expanded role for primary care in the follow-up of people with cancer is increasingly seen as critical for long-term sustainability of the health system in many developed nations. ${ }^{16}{ }^{17}$ This is recognised in UK's National Cancer Survivorship Initiative ${ }^{18}$ and, specifically in relation to prostate cancer, by the National Institute of Health and Care Excellence. ${ }^{19}$

A systematic review of primary care-based follow-up in trials with breast and colon cancer survivors found no statistically significant differences between primary and secondary care follow-up in terms of patient well-being, psychological morbidity and patient satisfaction. ${ }^{20} \mathrm{~A}$ randomised controlled trial of GP-led follow-up of people with melanoma found no significant difference in health status or anxiety and depression between intervention and control groups. However, there were significant improvements in some aspects of patient satisfaction with care for those receiving GP-led melanoma follow-up. ${ }^{21} \mathrm{~A}$ recent rapid review of the evidence reported on seven trials of shared care in cancer; most of these focused on increasing the primary care team's involvement in managing symptoms during or immediately following treatment for cancer. ${ }^{22} 23$ These trials found that shared care models of cancer follow-up can improve a range of important process outcomes including patient and provider satisfaction, provider confidence and knowledge and patient perceptions of care. No trials of shared care have tested a structured approach to sharing cancer surveillance, management of treatment-related effects and psychosocial support 
between hospital and primary care after completion of treatment. Furthermore, there are no trials reported to date of prostate cancer follow-up in primary care.

Previous trials of primary care follow-up have focused on detection of recurrence to the exclusion of the multiple needs and comorbidities of patients which may be more appropriately dealt with from a generalist perspective. $^{22} 24$ The key elements in a conceptual model of generalism include accessibility, holistic patient-centred, team-based care, care coordination, continuity and management of complex multiple problems. ${ }^{25}$ Evidence from previous studies with cancer survivors followed up in primary care suggest that they are more likely to receive preventive interventions for conditions other than cancer, whereas those followed up by oncologists are more likely to receive interventions directed at cancer surveillance. ${ }^{26}$ Primary care may therefore have an important broader generalist role to play in cancer follow-up.

\section{Principles underpinning a novel shared care model of follow-up for prostate cancer}

In 2005, the US Institute of Medicine (IOM) released a landmark report From Cancer Patient to Cancer Survivor: Lost in Transition. ${ }^{27}$ The report recommended that new research initiatives focused on follow-up of patients with cancer were urgently needed to guide effective survivorship care. The IOM report outlined four essential components of survivorship care planning: (1) prevention of recurrent and new cancers, and of other late effects; (2) surveillance for cancer spread, recurrence or second cancers; assessment of medical and psychosocial late effects; (3) interventions for consequences of cancer and its treatment and (4) coordination between specialists and primary care providers to ensure that all of the survivors' health needs are addressed.

The most common systemic problems in providing comprehensive cancer care include requirement for a case manager, local accessible health services and doctors who communicate with each other. ${ }^{28} \mathrm{~A}$ systematic review of guidelines for follow-up care in prostate cancer highlighted that most focus on the detection of cancer recurrence and assessment of the medical consequences of treatment, with little attention placed on identifying and responding to other key unmet needs. ${ }^{29}$

In the ProCare Trial we are applying the following principles to guide the design of a model of shared hospital and primary care for prostate cancer (figure 1).

\section{Communication between hospital and primary care}

A current major issue in cancer follow-up is coordination of care between specialists and general practice, partly due to out-dated approaches to communication. Timely and systematic communication between hospital and community care providers is urgently required to clarify the roles and responsibilities of all, including the person with cancer. ${ }^{30}$ 31 An Australian trial comparing methods of communication between hospital and general practices found that fax had higher receipt rates than post and was the most preferred method by GPs. ${ }^{32}$ An innovative trial of electronic faxing of standardised information to GPs about a patient's chemotherapy regime has shown that this approach led to improved GP confidence in managing adverse effects of treatment and increased satisfaction with shared care. ${ }^{33}$ Survivorship care plans (SCPs) are recommended as an important tool to facilitate communication and clarify responsibility during the transition from active treatment to survivorship. ${ }^{26}$ There has only been one trial of the use of SCPs in primary care ${ }^{34}$ but several methodological issues have been raised about this trial which may explain its negative findings ${ }^{35}$ hence further evidence is needed.

\section{Promotion of patient involvement and engagement}

Patients with cancer want to be involved with decisionmaking, and wish to participate in strategies to remain well. ${ }^{36}$ Involving patients with chronic diseases in their disease management results in better communication with physicians, improved self-reported health and reduced health distress, few hospitalisations and reduced health costs. ${ }^{37} 38$ Self-management approaches also have potential for ameliorating the functional and emotional problems experienced by prostate cancer survivors. ${ }^{39}$ A systematic review of patient activation approaches has shown they can alter the content of consultations and improve the identification of patients' concerns. ${ }^{40}$ Approaches that allow patients to list and share their concerns with their doctor, particularly if linked to practitioner interventions, showed particular promise in this review. A separate systematic review of problem checklists found that these can empower patients with cancer to ask relevant questions in healthcare consultations. ${ }^{41}$

\section{Tailoring care to specific needs of individual patients}

Cancer survivors have different needs. ${ }^{28} 42$ Therefore, interventions need to be systematically tailored to each individual. A review of tailored versus standardised information interventions in the health promotion area found that tailored interventions were significantly more effective in promoting health behaviour outcomes. ${ }^{43}$ A randomised controlled trial with 543 prostate and breast cancer survivors tested the efficacy of sequentially tailored versus standardised materials on improving diet and exercise behaviours and found that those receiving tailored materials had improved lifestyle behaviours. ${ }^{44}$

\section{Aims of the ProCare Trial}

The ProCare Trial is a phase II trial of a multifaceted intervention designed to: (1) be patient-centred by eliciting individual needs and assisting patients to direct their healthcare; (2) provide appropriate multidisciplinary referrals and tailored information to patients; (3) provide holistic care coordination by a GP to address the multifaceted physical, psychosexual and social needs of 
Figure 1 Trial flow chart.

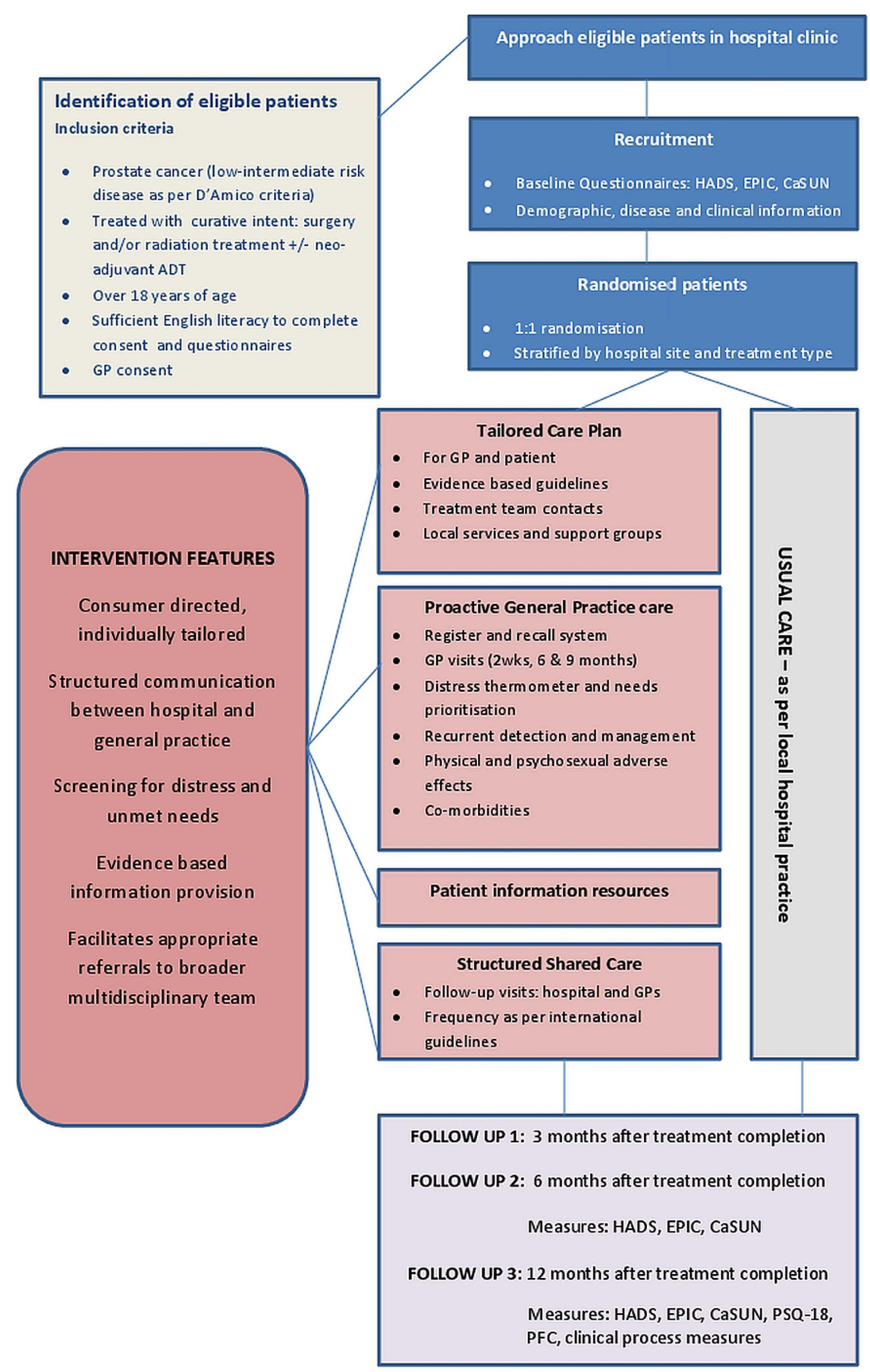

men with prostate cancer and (4) improve the timeliness and content of communication between hospital and primary care.

The trial is set within the Medical Research Council framework for the development and evaluation of complex interventions. ${ }^{45} 46$ The objectives of this phase II trial reflect the need to optimise the intervention, establish acceptability of the intervention and randomisation, confirm suitability of outcome measures and provide estimates of efficacy, and recruitment and attrition rates to allow planning of a larger phase III trial. It therefore does not specifically employ a statistical hypothesis-testing framework.
Methods and analysis

Trial design and randomisation

A phase I study that operationalised the different components of the intervention and explored clinical feasibility and acceptability has been completed. Eleven men who met the eligibility criteria were recruited from two hospitals in Perth, Western Australia, with all receiving the intervention and completing the outcome measures throughout the 12 months of follow-up. Participants were interviewed by telephone after each of their three GP visits, with the interview data demonstrating acceptability of the intervention. Issues pertaining to the intervention and the outcome 
measures have been addressed and incorporated into the phase II trial.

The phase II trial is a multisite randomised controlled trial. Men who meet the eligibility criteria and who consent to participate are randomised 1:1 to either usual care (control arm) or to trial shared care (intervention arm). Randomisation is being performed using a centralised independent tele-randomisation system managed by the National Health and Medical Research Council (NHMRC) Clinical Trials Centre, based at the University of Sydney. Stratifying variables for randomisation are hospital site and treatment type.

\section{Population and setting}

Men are being recruited from one rural and three urban public treatment centres in two Australian states (Western Australia and Victoria); private patients are also being recruited from one centre in Victoria.

\section{Inclusion criteria}

1. Pathologically confirmed prostate cancer.

2. Completed surgery and/or radiotherapy (brachytherapy or external beam, and which may also include neoadjuvant androgen deprivation therapy) with curative intent; study entry within 8 weeks postoperatively or 3 weeks after completion of radiotherapy.

3. Able to read and write English at a level sufficient to give informed consent and complete study procedures including written questionnaires without an interpreter.

4. Have a GP who agrees to participate in the trial.

\section{Exclusion criteria}

1. Suspicion or evidence of metastatic disease.

2. Severe psychiatric or cognitive disorder, which in the opinion of the investigator would compromise participation the study.

3. Treatment with palliative intent.

4. No GP.

5. Patients with a pathologically confirmed diagnosis of prostate cancer with any of the following high risk features (cT3; PSA $>20$ or Gleason score $>8$ ).

6. Patients having androgen deprivation therapy following radiotherapy, irrespective of risk level.

Minimal data will be completed with consent from eligible men who decline to participate to measure selection bias.

\section{Participant and GP recruitment procedures}

Men receiving radiotherapy treatment are approached about the trial towards the end of their treatment, while men having surgery are approached once their histopathology results are confirmed. If men consent to participate, their GP is faxed trial information and a consent form. If their GP agrees to participate, the patient is formally enrolled in the trial and randomised. If the GP declines, the patient receives standard hospital follow-up care outside the trial. GPs are eligible to have more than one patient in the trial regardless of treatment allocation.

\section{Intervention}

The intervention is based on a shared care model where two of the five routine hospital visits during the first 12 months of follow-up are replaced by GP visits. An additional GP visit shortly after the completion of their treatment for prostate cancer is intended to re-engage the patient with their GP (tables 1 and 2).

In addition to the altered schedule of follow-up, the following specific components of the intervention are designed to support the model of shared care:

1. Structured systematic communication, using an SCP.

2. GP clinical management guidelines.

3. Register and recall system for follow-up appointments.

4. Screening for distress and unmet needs using the distress thermometer (DT) and problem checklist. ${ }^{47}$

5. Provision of patient information resources.

\section{Survivorship care plan}

A tailored SCP using information from the patient's hospital notes is developed at the end of treatment by a member of the research team. It is produced using an electronic template and includes information on: prostate cancer diagnosis and treatment history; treatment team and contact details for rapid access and advice; the schedule of follow-up visits and tests for recurrence; early and later side effects of treatment applicable to treatment modality; information on relevant local services and resources including the Cancer Council Helpline, prostate cancer support groups, and stress management and relaxation programmes.

A draft of the care plan is discussed with the patient by telephone by one of the research team before their initial GP visit allowing additional information to be incorporated such as current adverse effects of treatment. The finalised care plan is provided to the patient, their GP and hospital specialist. The care plan is faxed to the GP before the first follow-up visit and is designed to be incorporated into the patient's GP medical record.

\section{GP management guidelines}

GP management guidelines, based on international and local guidelines, ${ }^{48}$ are included in the GP's copy of the care plan. They include guidelines on frequency of PSA testing and digital rectal examination to detect and manage recurrence, management of common physical and psychosexual adverse treatment effects, interpretation of the DT, and referral information to relevant services (eg, sexual health and continence services).

\section{Register and recall system}

This is a well-established component of good chronic disease management to reduce loss to follow-up and implement timely care. ${ }^{50} \mathrm{~A}$ reminder letter is sent by the research team to the patient to attend each follow-up appointment, either at the hospital or general 
Table 1 Frequency of follow-up visits in control and intervention arms (surgery and radiotherapy, and radiotherapy only)

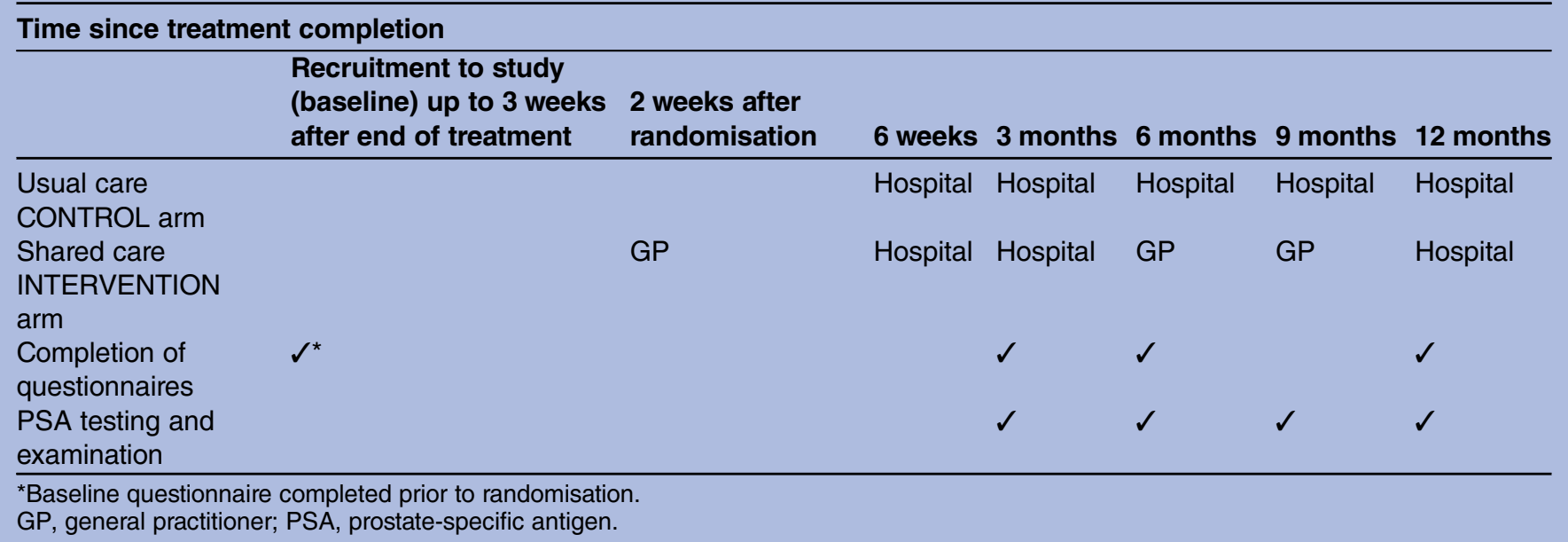

practice. Reminder letters are sent to GPs before the 6-month and 9-month visits.

\section{Screening for distress and concerns}

The DT is a widely used validated screening tool for assessing psychological distress in people affected by cancer. ${ }^{47}$ Men complete the DT on the day of each GP visit and GPs are advised to explore the meaning of distress and consider depression or anxiety in men with a cut-off score of four or greater. A modified problem checklist, specific to prostate cancer, has been incorporated into the DT, and covers physical and psychosocial issues. Men are asked to tick any problems they have experienced in the previous week, identifying the three most important. They give the checklist to their GP at the beginning of the consultation, to shape the content of the consultation and to facilitate discussion of specific unmet needs.

\section{Patient information resources}

In addition to the information within the SCP, patients are offered the following prostate cancer specific information, according to their specific circumstances:

- Localised prostate cancer: a guide for men and their families (Cancer Council Australia 2010, 4th edition);
- Continence and prostate: a guide for men undergoing prostate surgery (Continence Foundation of Australia, 2008);

- Treat ED: prostate edition. Understanding the impact of prostate cancer treatment on erectile function (Eli Lilly Australia);

- Maintaining your well-being: information on depression and anxiety for men with prostate cancer and their partners (beyond blue in association with Prostate Cancer Foundation of Australia).

\section{Control group}

Men in the control group receive clinical care according to current hospital practice with frequency of visits as outlined in tables 1 and 2, consistent with current international guidelines. ${ }^{49}$

\section{Outcomes and measures}

As a phase II trial we have not determined a single primary outcome measure but instead are applying a battery of established instruments to measure the effects of the various components of this complex intervention. ${ }^{46}$ This will inform decisions about outcome measures for a future phase III trial.

Table 2 Frequency of follow-up visits in control and intervention arms (surgery only)

\section{Time since treatment completion}

\begin{tabular}{|c|c|c|c|c|c|c|}
\hline & $\begin{array}{l}\text { Recruitment to study } \\
\text { (baseline) up to } \\
8 \text { weeks postsurgery }\end{array}$ & $\begin{array}{l}2 \text { weeks after } \\
\text { randomisation }\end{array}$ & 3 months & 6 months & 9 months & 12 months \\
\hline Usual care CONTROL arm & & & Hospital & Hospital & Hospital & Hospital \\
\hline $\begin{array}{l}\text { Shared care INTERVENTION } \\
\text { arm }\end{array}$ & & GP & Hospital & GP & GP & Hospital \\
\hline Completion of questionnaires & $\boldsymbol{S}^{*}$ & & $\checkmark$ & $\checkmark$ & & $\checkmark$ \\
\hline PSA testing and examination & & & $\checkmark$ & $\checkmark$ & $\checkmark$ & $\checkmark$ \\
\hline
\end{tabular}


Demographics and clinical variables include age, postcode, marital status, education level and occupation, treatment type, diagnosis, stage of disease and patient reported comorbidities.

\section{Patient-reported outcome measures}

Psychological distress

Hospital Anxiety and Depression Scale (HADS) ${ }^{51}$-this 14-item scale has been widely used to measure distress in people with cancer; it has been extensively validated and shown to perform well in a wide range of populations (Cronbach $\alpha=0.82$; sensitivity and specificity 0.80$).{ }^{52}$ A systematic review of measures of distress in patients with cancer has concluded that the HADS performs better than other similar measures. ${ }^{53}$

\section{Survivors' unmet needs}

Cancer Survivors' Unmet Needs measure (CaSUN)-this 35-item scale assesses unmet needs across information, patient care, psychosocial, physical and sexual domains. ${ }^{54}$ The scale has good acceptability, internal consistency (Cronbach $\alpha=0.96$ ) and construct validity. Owing to difficulties with the response format experienced by some participants in the phase I study, a simplified four-point response format is being used in this trial (no, low, moderate and high need).$^{55}$

\section{Quality of life}

Expanded Prostate Cancer Index Composite (EPIC) assesses prostate-specific quality of life (32 items with 4 subscales: urinary, bowel, sexual and hormonal function). It has greater coverage of key domains and sensitivity to treatment effects than previous prostate-specific quality of life measures. ${ }^{56}$ It shows good test-retest reliability and internal consistency for all domain summary scores (each $\mathrm{r}>0.80$ and Cronbach $\alpha>0.82$ ).

The Short-form Patient Satisfaction Questionnaire (PSQ-18) consists of 18 items covering access, convenience, continuity, perceived communication between healthcare providers and technical competence. ${ }^{57}$ It shows good internal consistency (each Cronbach $\alpha>0.7$ ) and strong correlations with the original 50-item PSQ III. ${ }^{58}$ After piloting in the phase I study, this scale has been modified to refer explicitly to the cancer follow-up care provided by hospital doctors and GPs during the previous 12 months.

Preference for Follow-up Care (PFC)—questions about preferences for future follow-up care have been adapted from the Cancer Survivors Follow-up Care Study (Adult Survivors Survey; personal communication A Girgis). A direct question about preference for specific type of follow-up care has also been included.

Participants complete the HADs, CaSUN and EPIC at four time points: prior to randomisation and then at 3 , 6 and 12 months of follow-up. Participants complete the PSQ-18 and PFC after their 12-month follow-up appointment.

\section{Clinical process measures}

The following clinical information will be collected from GP medical records and Medicare Australia data, including Medical Benefits Schedule (MBS) and Pharmaceutical Benefits Schedule (PBS) data:

A. Recurrence rates and detection: use of PSA according to protocol ${ }^{49}$ and time to detect recurrence.

B. Mental healthcare: for example, prescribing of antidepressants; referrals to clinical psychologists and use of specific Medicare Mental Health Care Plan items.

C. Detection and management of psychosexual adverse effects: for example, prescribing of phosphodiesterase type- 5 inhibitors and referrals to sexual health services.

D. Detection and management of other physical adverse effects of treatment: prescribing prespecified drugs for urinary and bowel symptoms (eg, oxybutynin, prazosin, loperamide, steroid enemas) and referrals to continence physiotherapy or urology.

E. Management of comorbidities will be determined by pathology data for common tests performed in the management of common chronic disease (eg, vascular disease and diabetes) and will include, for example, lipids and glycated hemoglobin. This is to assess whether the model of shared care has an effect on the management of other comorbidities.

Health care resource usage — data will be collected regarding hospitalisations, visits to healthcare professionals, investigations and medications, predominantly through Medicare Australia (MBS and PBS) and GP record audit. Unit costs obtained from a variety of sources (eg, Australian refined diagnosis-related groups, MBS and PBS) will be applied to the resource usage data collected within the trial to estimate the incremental cost of the shared care model versus standard care from a health service perspective.

Trial feasibility-as a phase II trial we will obtain data on patient eligibility, recruitment and attrition rates, GP recruitment and attrition rates, and response rates to outcome measures to inform decisions and planning for a larger phase III trial.

\section{Sample size}

The study is designed to provide preliminary estimates of the feasibility and the efficacy of the shared care intervention for phase III planning purposes and does not employ a statistical hypothesis testing framework. The sample size is based on ensuring adequate information is collected to yield preliminary estimates of the treatment effect and of between-patient variation that are sufficiently precise for phase III trial planning purposes. The sample size target was revised at a steering committee meeting on 2 August 2012. This was in response to lower accrual rates than predicted, specifically due to a lower proportion of low-moderate risk prostate cancers than originally estimated. The revised target of 90 men was selected to ensure that the $95 \%$ CIs for the mean difference between the two groups on the patient 
reported outcome measures would extend no further than \pm 0.5 of a SD with $80 \%$ probability and allowing for $10 \%$ attrition at 12 months (ie, complete data on $\mathrm{N}=80$ is required). This level of precision corresponds to what has been proposed as a minimal clinically important difference of health-related quality of life measures ${ }^{59}$ allowing us to identify clinically significant harm from the intervention if it existed. Data from a trial of a groupbased intervention involving 331 men with prostate cancer in Victoria, Australia (ACTRN12606000184572) has been used to estimate how this level of precision will translate to estimates from the HADS and EPIC instruments. Recruitment was completed in July 2013.

\section{Analyses}

Baseline characteristics of the two arms will be described. Possible attrition bias will be assessed by comparing non-completion rates between treatment groups in conjunction with the baseline characteristics of those who withdraw or die against those who remain in the study.

\section{Estimating potential effect size and coefficient of variation}

Mean scores of HADS, CaSUN, EPIC and PSQ-18 will be compared between intervention and usual care groups. Mean differences between groups will be calculated with 95\% CIs at each follow-up time point with and without adjusting for baseline score, site and treatment type (surgery and/or radiotherapy). Treatment groups will be compared on the categorical endpoints (eg, clinical process measures) using $\chi^{2}$ tests. Logistic regression modelling will also be undertaken to estimate the treatment effect on these endpoints adjusting for baseline covariates. The principal emphasis of the analysis will be on obtaining estimates of the treatment effect size and assessing feasibility in order to inform the design of a subsequent phase III trial. $p$ Values for the multiple comparisons between the groups will be interpreted in this context.

\section{DISCUSSION}

The ProCare Trial has several novel elements: it is the first randomised controlled trial of a model of shared care for men with prostate cancer; it is the first trial to use the DT in primary care and the first to test a specific problem checklist to identify unmet needs of cancer survivors in primary care. We are testing SCP in primary care. One of the problems with the Grunfeld trial of SCPs in primary care was the high proportion of prevalent cancer cases who had completed treatment several years previously, and were possibly less likely to benefit. ${ }^{34}{ }^{35}$ We are therefore only recruiting men who have very recently completed their cancer treatment and in their first 12 months of follow-up care.

As a phase II trial it is designed to yield estimates of sufficient precision for phase III trial planning purposes. However, as with all trials of alternative models of cancer follow-up, an outstanding methodological issue is the selection of an appropriate primary outcome measure. Most trials have measured satisfaction with care and a range of health-related quality of life measures, finding no differences between hospital and primary care follow-up. ${ }^{22}$ Trials in populations at low risk of cancer recurrence would need to be unfeasibly large to detect differences in survival. The ProCare Trial includes a range of outcome measures including disease-specific quality of life and unmet needs. The intervention is designed to improve the identification of unmet needs and implement best practice management in the expectation that this will improve disease-specific quality of life and overall well-being.

We are recruiting men from a range of metropolitan and rural settings in two states in Australia including public and private healthcare settings. Based on discussions with urologists and radiation oncologists we have chosen only to recruit men with low-intermediate risk of disease recurrence, based on the D'Amico criteria. ${ }^{60}$ As the first trial of shared follow-up care in prostate cancer it was agreed by the investigator team for safety reasons to focus initially on men with low-intermediate risk disease. This is also consistent with international approaches to risk stratified follow-up. ${ }^{61}$ Our trial population is likely to be representative of a wide range of men with lowintermediate risk prostate cancer who might be offered alternative follow-up arrangements if this model of care were shown to be feasible and acceptable.

We plan to complete follow-up in July 2014 and report trial results in early 2015 .

\section{Dissemination}

This is the first randomised controlled trial of a model of shared care for men with prostate cancer; it is also the first trial to use the DT in primary care and the first to test a specific checklist to identify unmet needs of cancer survivors in primary care. We plan to publish the main trial outcomes in a single paper and anticipate publishing additional papers exploring the data in more detail and relating to the implementation of this complex intervention. We will also present the findings at national and international conferences from late 2014.

\section{Author affiliations}

${ }^{1}$ General Practice and Primary Health Care Academic Centre, The University of Melbourne, Carlton, Victoria, Australia

${ }^{2}$ Department of General Practice, School of Primary, Aboriginal and Rural Health Care, The University of Western Australia, Perth, Western Australia,

Australia

${ }^{3}$ Department of Medical Oncology, Peter MacCallum Cancer Centre, East Melbourne, Victoria, Australia

${ }^{4}$ Psycho-oncology Co-operative Research Group (PoCoG), School of Psychology, The University of Sydney, Sydney, New South Wales, Australia

${ }^{5}$ School of Surgery, The University of Western Australia, Western Australia, Australia

${ }^{6}$ Urology Department, Fremantle Hospital, Fremantle, Western Australia, Australia

${ }^{7}$ NHMRC Clinical Trials Centre, Sydney Medical School, The University of Sydney, Sydney, New South Wales, Australia

${ }^{8}$ School of Public Health, The University of Sydney, Sydney, New South Wales, Australia

${ }^{9}$ Genesis Cancer Care, Royal Perth Hospital, Perth, Western Australia, Australia 
${ }^{10}$ Prostate Cancer Foundation of Australia, Perth, Western Australia, Australia

${ }^{11}$ Department of Cancer Experiences Research, Peter MacCallum Cancer

Centre, East Melbourne, Victoria, Australia

${ }^{12}$ Urology Department, Royal Perth Hospital, Perth, Western Australia, Australia

${ }^{13}$ Department of Radiation Oncology, Peter MacCallum Cancer Centre, East Melbourne, Victoria, Australia

${ }^{14}$ Department of Surgery, Faculty of Medicine, Nursing and Health Sciences, Monash University, Melbourne, Victoria, Australia

Acknowledgements This trial is supported by the Cancer Australia Primary Care Collaborative Cancer Clinical Trials Group (PC4) and the Australian and New Zealand Urogenital and Prostate Cancer Trials Group (ANZUP). The authors thank the following people who have also been involved in the establishment and conduct of this trial: Sharon Ray, Sema Cakan, Anna Ugalde, Rose Kamateros, Emily Habgood, Danielle Miller and Shona Silvester. We are grateful to all the trial participants, general practitioners (GPS) and hospital specialists involved in the trial.

Contributors JE, JD, AM, MJ, MK, MP, AM and LT coordinated the design, funding award and implementation of the study and led the writing of the manuscript. DH contributed to the study design, funding award and implementation of the study in Western Australia and contributed to the writing of the manuscript. TL provided clinical expertise. RC provided expertise from a consumer perspective for study design, funding award and implementation of the study. $\mathrm{CH}$ undertook recruitment and data collection at Fremantle Hospital, Western Australia. AmH undertook recruitment and data collection for Victorian sites and approved the final manuscript. AkH, JV, SG and MF approved the final manuscript. PS contributed to the study design, funding award and implementation of the study in Victoria and to the writing of the manuscript.

Funding This trial is supported by the National Health and Medical Research Council (grant ID 1003414). Associate Professor Schofield and Associate Professor Pirotta are supported by National Health and Medical Research Council Career Development Fellowships.

Competing interests None. TL provided clinical expertise and was principal investigator at Royal Perth Hospital, AkH was principal investigator at Royal Perth Hospital Urology Services, Western Australia, recruitment site, JV was principal investigator at Peter MacCallum Cancer Centre, Sunshine Hospital, Victoria and SG was principal investigator at Peter MacCallum Cancer Centre, Bendigo Hospital, Victoria

Ethics approval Ethics approval has been granted from the University of Western Australia's Human Research Ethics Committee (RA/4/1/4447) as well as from all hospital recruitment sites in Western Australia and Victoria. The study has also been approved by the External Review Committee of the Commonwealth Department of Human Services to obtain Medicare Benefits Schedule and Pharmaceutical Benefits Schedule data from participants with their consent.

Provenance and peer review Not commissioned; peer reviewed for ethical and funding approval prior to submission.

Open Access This is an Open Access article distributed in accordance with the Creative Commons Attribution Non Commercial (CC BY-NC 3.0) license, which permits others to distribute, remix, adapt, build upon this work noncommercially, and license their derivative works on different terms, provided the original work is properly cited and the use is non-commercial. See: http:// creativecommons.org/licenses/by-nc/3.0/

\section{REFERENCES}

1. Center MM, Jemal A, Lortet-Tieulent J, et al. International variation in prostate cancer incidence and mortality rates. Eur Urol 2012;61:1079-92.

2. Ferlay J, Shin HR, Bray F, et al. Estimates of worldwide burden of cancer in 2008: GLOBOCAN 2008. Int J Cancer 2010;127:2893-917.

3. Cancer in Australia: an overview. Canberra: Australian Institute of Health and Welfare, 2012.

4. Cancer incidence projections: Australia, 2011-2020. Canberra: Australian Institute of Health and Welfare, 2012.
5. Smith BD, Smith GL, Hurria A, et al. Future of cancer incidence in the United States: burdens upon an aging, changing nation. J Clin Oncol 2009;27:2758-65.

6. SEER Stat Fact Sheets: Prostate Cancer: National Cancer Institute, 2013.

7. O'Brien R, Rose PW, Campbell C, et al. Experiences of follow-up after treatment in patients with prostate cancer: a qualitative study. BJU Int 2010;106:998-1003.

8. Boberg E, Gustafson D, Hawkins R, et al. Assessing the unmet information, support and care delivery needs of men with prostate cancer. Patient Educ Couns 2003;49:233-42.

9. Ream E, Quennell A, Fincham L, et al. Supportive care needs of men living with prostate cancer in England: a survey. Br J Cancer 2008;98:1903-9.

10. Resnick MJ, Koyama T, Fan K-H, et al. Long-term functional outcomes after treatment for localized prostate cancer. $N$ Engl J Med 2013;368:436-45.

11. Cockle-Hearne J, Charnay-Sonnek F, Denis L, et al. The impact of supportive nursing care on the needs of men with prostate cancer: a study across seven European countries. Br J Cancer 2013;109:2121-30.

12. Smith DP, Supramaniam R, King MT, et al. Age, health, and education determine supportive care needs of men younger than 70 years with prostate cancer. J Clin Oncol 2007;25:2560-6.

13. Smith DP, King MT, Egger S, et al. Quality of life three years after diagnosis of localised prostate cancer: population based cohort study. BMJ 2009;339:b4817.

14. Khan NF, Watson E, Rose PW. Primary care consultation behaviours of long-term, adult survivors of cancer in the UK. $\mathrm{Br} J$ Gen Pract 2011;61:197-9.

15. Heins MJ, Korevaar JC, Rijken PM, et al. For which health problems do cancer survivors visit their General Practitioner? Eur J Cancer 2013;49:211-18.

16. Role Redesign Primary Care-Shared Care. Models of Cancer Treatment and Surveillance Post Treatment. Final Report. . In: Communications. GCa, editor. Canberra: Cancer Australia, 2010.

17. Pascoe SW, Neal RD, Allgar VL, et al. Psychosocial care for cancer patients in primary care? Recognition of opportunities for cancer care. Fam Pract 2004;21:437-42.

18. Richards M, Corner J, Maher J. The National Cancer Survivorship Initiative: new and emerging evidence on the ongoing needs of cancer survivors. Br J Cancer 2011;105(Suppl 1):S1-4.

19. Guideline development group. Prostate cancer: diagnosis and treatment. London: National Institute of Clinical Excellence, 2008.

20. Lewis RA, Neal RD, Williams $\mathrm{NH}$, et al. Follow-up of cancer in primary care versus secondary care: systematic review. $\mathrm{Br} J$ Gen Pract 2009;59:e234-47.

21. Murchie $P$, Nicolson MC, Hannaford PC, et al. Patient satisfaction with GP-led melanoma follow-up: a randomised controlled trial. $\mathrm{Br} J$ Cancer 2010;102:1447-55.

22. Emery JD, Trevena L, Mazza D, et al. The role of primary and community-based healthcare professionals in early detection and follow-up of cancer: a rapid review of best practice models. Sydney, NSW: Sax Institute, 2013.

23. Emery JD, Shaw K, Williams B, et al. The role of primary care in early detection and follow-up of cancer. Nat Rev Clin Oncol 2014;11:38-48.

24. Taggart F, Donnelly P, Dunn J. Options for early breast cancer follow-up in primary and secondary care-a systematic review. BMC Cancer 2012;12:238.

25. Gunn JM, Palmer VJ, Naccarella L, et al. The promise and pitfalls of generalism in achieving the Alma-Ata vision of health for all. Med $J$ Aust 2008;189:110-12.

26. Grunfeld E, Earle C. The Interface Between Primary and Oncology Speciality Care: Treatment Through Survivorship. J Natl Cancer Inst Monogr 2010;2010:25-30.

27. Hewitt M, Greenfield S, Stovall E. From cancer patient to cancer survivor: lost in transition. Washington, DC, 2006.

28. Hodgkinson $\mathrm{K}$, Butow $\mathrm{P}$, Hobbs $\mathrm{KM}$, et al. After cancer: the unmet supportive care needs of survivors and their partners. J Psychosoc Oncol 2007;25:89-104.

29. Mclntosh HM, Neal RD, Rose $\mathrm{P}$, et al. Follow-up care for men with prostate cancer and the role of primary care: a systematic review of international guidelines. Br J Cancer 2009;100:1852-60.

30. Jiwa M, Saunders CM, Thompson SC, et al. Timely cancer diagnosis and management as a chronic condition: opportunities for primary care. Med J Aust 2008;189:78-82.

31. Walsh J, Harrison JD, Young JM, et al. What are the current barriers to effective cancer care coordination? A qualitative study. BMC Health Serv Res 2010;10:132.

32. Chen Y, Brennan N, Magrabi F. Is email an effective method for hospital discharge communication? A randomized controlled trial to 
examine delivery of computer-generated discharge summaries by email, fax, post and patient hand delivery. Int $J$ Med Inform 2010;79:167-72

33. Jefford M, Baravelli C, Dudgeon P, et al. Tailored chemotherapy information faxed to general practitioners improves confidence in managing adverse effects and satisfaction with shared care: results from a randomized controlled trial. J Clin Oncol 2008;26:2272-7.

34. Grunfeld E, Julian J, Pond G, et al. Evaluating survivorship care plans: results of a randomized, clinical trial of patients with breast cancer. J Clin Oncol 2011;29:4755-62.

35. Jefford M, Schofield P, Emery J. Improving survivorship care. J Clin Oncol 2012;30:1391-2.

36. Brown RF, Butow PN, Sharrock MA, et al. Education and role modelling for clinical decisions with female cancer patients. Health Expect 2004;7:303-16.

37. Barlow J, Wright C, Sheasby J. Self-management approaches for people with chronic conditions: a review. Patient Educ Couns 2002;48:177-87.

38. Deadman J, Leinster S, Owens R. Taking responsibility for cancer treatment. Soc Sci Med 2001;53:669-77.

39. Cockle-Hearne J, Faithfull S. Self-management for men surviving prostate cancer: a review of behavioural and psychosocial interventions to understand what strategies can work, for whom and in what circumstances. Psychooncology 2010;19:909-22.

40. Griffin SJ, Kinmonth AL, Veltman MW, et al. Effect on health-related outcomes of interventions to alter the interaction between patients and practitioners: a systematic review of trials. Ann Fam Med 2004;2:595-608.

41. Dimoska A, Tattersall MH, Butow PN, et al. Can a 'prompt list' empower cancer patients to ask relevant questions? Cancer 2008;113:225-37.

42. Jefford M, Karahalios A, Angle A. Understanding issues for cancer survivors: informing the development of a written information booklet. Psychooncology 2007;16:S273.

43. Ryan P, Lauver DR. The efficacy of tailored interventions. J Nurs Scholarsh 2002;34:331-7.

44. Demark-Wahnefried W, Clipp EC, Lipkus IM, et al. Main outcomes of the FRESH START trial: a sequentially tailored, diet and exercise mailed print intervention among breast and prostate cancer survivors. J Clin Oncol 2007;25:2709-18.

45. Developing and evaluating complex interventions: new guidance. London: Medical Research Council, 2008.

46. Campbell N, Murray E, Darbyshire J, et al. Designing and evaluating complex interventions to improve healthcare. BMJ 2007;334:455-9.
47. Jacobsen $\mathrm{P}$, Donovan K, Trask P. Screening for psychologic distress in ambulatory care patients. Cancer 2005;103:1494-502.

48. Localised prostate cancer: a guide for men and their families. Andrology Australia \& Australian Prostate Cancer Collaboration, 2009.

49. Heidenreich A, Bolla M, Joniau S, et al. Guidelines on prostate cancer. European Association of Urology, 2009.

50. Wagner EH, Austin BT, Davis $\mathrm{C}$, et al. Improving chronic illness care: translating evidence into action. Health Aff 2001;20:64-78.

51. Zigmond A, Snaith R. The Hospital Anxiety and Depression Scale. Acta Psychiatr Scand 1983;67:361-70.

52. Bjellanda I, Dahlb A, Haugc T, et al. The validity of the Hospital Anxiety and Depression Scale. An updated literature review. $J$ Psychosom Res 2002;52:69-77.

53. Luckett $\mathrm{T}$, Butow $\mathrm{PN}$, King MT, et al. A review and recommendations for optimal outcome measures of anxiety, depression and general distress in studies evaluating psychosocial interventions for English-speaking adults with heterogeneous cancer diagnoses. Support Care Cancer 2010;18:1241-62.

54. Hodgkinson K, Butow P, Hunt GE, et al. The development and evaluation of a measure to assess cancer survivors' unmet supportive care needs: the CaSUN (Cancer Survivors' Unmet Needs measure). Psychooncology 2007;16:796-804.

55. Schofield P, Gough K, Lotfi-Jam K, et al. Validation of the Supportive Care Needs Survey-short form 34 with a simplified response format in men with prostate cancer. Psychooncology 2012;21:1107-12.

56. Wei JT, Dunn RL, Litwin MS, et al. Development and validation of the expanded prostate cancer index composite (EPIC) for comprehensive assessment of health-related quality of life in men with prostate cancer. Urology 2000;56:899-905.

57. Marshall G, Hays R. The patient satisfaction questionnaire short-form (PSQ-18). RAND Corporation, 1994.

58. Ware JE Jr, Davies-Avery A, Stewart AL. The measurement and meaning of patient satisfaction. Health Med Care Serv Rev 1978;1:1, 3-15.

59. Norman GR, Sloan JA, Wyrwich KW. Interpretation of changes in health-related quality of life: the remarkable universality of half a standard deviation. Med Care 2003;41:582-92.

60. D'Amico AV. Combined-modality staging for localized adenocarcinoma of the prostate. Oncology 2001;15:1049-59; discussion 60-2, 64-5, 69-70, 73-5.

61. Watson EK, Rose P, Neal RD, et al. Personalised cancer follow-up: risk stratification, needs assessment or both? Br J Cancer 2012;106:1-5. 